Publ. RIMS, Kyoto Univ.

Vol. 6 (1970), 43-70

\title{
Uniqueness in Cauchy's Problem for Certain Fourth Order Elliptic Equations
}

By

\author{
Kazunari HAYASHIDA*
}

1. We consider in the $N$ dimensional space $R^{N}$ witl coordinate $\left(x_{1}, \cdots, x_{N}\right)$ and with norm $r\left(=\left(\sum_{i=1}^{N} x_{i}^{2}\right)^{1 / 2}\right)$. Let $\Gamma$ be a smooth initial hypersurface containing the origin in its interior and let $\Omega$ be a domain whose boundary contains $\Gamma$. We consider the real elliptic operator of the form

$$
L=a_{i j} \frac{\partial^{2}}{\partial x_{i} \partial x_{j}}+b_{i} \frac{\partial}{\partial x_{i}}+c
$$

where $a_{i j} \in C^{1+\alpha}(\bar{\Omega})(\alpha>0)$ and $b_{i}, c \in L^{\infty}(\bar{\Omega})$.

When $L$ is of the form (1.1), uniqueness in Cauchy's problem for solutions of $L u=0$ was shown by several mathematicians (see cf. [1], $[4],[5],[7],[9],[16])$. On the other hand, when the coefficients of $L$ in (1.1) are smooth, Landis [12] and Lavrentév [13] proved that any solution $u$ of $L u=0$ satisfying the following two conditions vanishes identically in $\Omega:^{1)}$

(i) $u \in C^{2}(\bar{\Omega})$ and $L u=0$ in $\Omega$,

(ii) $u, u_{x_{i}}=o\left(\exp \left(-r^{-\delta}\right)\right)(r \rightarrow 0)$ along $\Gamma$,

where $\delta$ is a positive constant depending only on $L$ and $\Gamma$. Their method may be said to give an explicit estimate expressing a relation between the solution and the Cauchy data.

In this note we shall give another proof for their results. Our method will be applied to the elliptic system

Received November 5, 1969.

Communicated by S. Matsuura.

* Department of Mathematics, Nagoya University.

1) Previously Mergelyan [14] proved this result for harmonic functions. 


$$
L_{p} u_{p}=F_{p}\left(x, u_{q}, u_{q x_{i}}\right) \quad p=1, \cdots, m,
$$

where each $L_{p}$ is the elliptic operator of the form (1.1) and each nonlinear part $F_{p}$ satisfies

$$
\left|F_{p}\left(x, u_{q}, u_{q x_{i}}\right)-F_{p}\left(x, v_{q}, v_{q x_{i}}\right)\right| \leqq \text { const } \sum_{q=1}^{m} \sum_{|\alpha| \leq 1}\left|D^{\alpha}\left(u_{q}-v_{q}\right)\right|_{0}{ }^{2)}
$$

Theorem 1. There is a positive number $\delta$ such that if the solutions $\left\{u_{p}\right\}$ and $\left\{v_{p}\right\}$ of (1.2) satisfy

$$
u_{p}, v_{p} \in C^{2}(\bar{\Omega}) \cap C^{3}(\Gamma)
$$

and

$$
\begin{gathered}
u_{p}-v_{p}, u_{p x_{i}}-v_{p x_{s}}=o\left(\exp \left(-r^{-2 \delta}\right)\right) \quad(r \rightarrow 0) \quad \text { aiong } \Gamma \\
(p=1, \cdots, m, \quad i=1, \cdots, N),
\end{gathered}
$$

then $u_{p} \equiv v_{p}$ in $\Omega$, where $\delta$ depends oniy on $\left\{L_{p}\right\}$ and $\Gamma_{\text {. }}$

Next we treat the solution $u$ of the fourth order elliptic differential inequalities

$$
\left|L_{1} L_{2} u\right| \leqq \text { const } \sum_{|\alpha| \leq 3}\left|D^{\alpha} u\right|,
$$

where $L_{1}, L_{2}$ are of the form (1.1) whose coefficients are sufficiently smooth and the nonlinear part of the right of (1.4) satisfies the Lipschitz condition as in (1.3). Uniqueness for solutions of (1.4) was shown by several mathematicians (see cf. [10], [11], [15], [17], [18], $[19])$. In particular, Mizohata $[15]^{3)}$ proved that the solution $u \in C^{4}(\bar{\Omega})$ of (1.4) vanishes identically in $\Omega$ if the Cauchy data of $u$ vanishes on $\Gamma$. He used the singular integral method developed by Calderón [3]. On the other hand, Pederson [17], Protter [18] and Shirota [19] proved that the solution $u \in C^{4}(\bar{\Omega})$ of (1.4) vanishes identically in $\Omega$ if $D^{\alpha} u$ $(|\alpha| \leqq 3)$ tends to zero rapidly at an interior point of $\Omega$. They used an integral estimate with a weight function having singularity at the point.

Now does the solution of (1.4) vanish identically, if its Cauchy

2) We write $\frac{\partial^{|\alpha|}}{\partial x_{1} \alpha_{1} \cdots \partial x_{n} \alpha_{n}}=D^{\alpha}$ for any vector $\alpha=\left(\alpha_{1}, \cdots, \alpha_{n}\right)$.

3) He proved also for the more general case of fourth order elliptic equations. 
data tend to zero rapidly at a point? We shall answer to this problem, that is, we can prove

Theorem 2. There is a positive constant $\delta$ depending only on $L_{1}, L_{2}$ and $\Gamma$ such that if the solution $u \in C^{4}(\bar{\Omega}) \cap C^{7}(\Gamma)$ of (1.4) satisfies

$$
D^{\alpha} u=o\left(\exp \left(-r^{-2 \delta}\right)\right) \quad(r \rightarrow 0,|\alpha| \leqq 3)
$$

along $\Gamma$, then $u$ vanishes identically in $\Omega$.

Remark. If $\Gamma$ is a spherical surface in the neighborhood of the origin and if $L_{1}$ (or $L_{2}$ ) is the Laplacian operator at the origin, then the constant $\delta$ in Theorem 2 can be taken as $\delta>\mathbb{N}$.

2. Let us denote by $S_{d}$ an open sphere with the center $(d / 2,0$, $\cdots, 0)$ and with the radius $d / 2$. We put $\Omega_{h}=\left\{0<x_{1}<h\right\} \cap S_{1}, \Gamma_{h}=\{0$ $\left.<x_{1}<h\right\} \cap \partial S_{1}$ and $l_{h}=\left\{x_{1}=h\right\} \cap S_{1}$. In this section we shall prove the following

Proposition 2.1. If $u \in C^{n}\left(\bar{\Omega}_{a}\right) \cap C^{2 m-1}\left(\Gamma_{a}\right)$ and $D^{\alpha} u=o$ (exp $\left.\left(-r^{-2 \delta-\varepsilon}\right)\right)(r \rightarrow 0,|\alpha| \leqq m-1)$ along $\Gamma_{a}$ for any fixea positive numbers $\delta$ and $\varepsilon$, then there is a function $v$ such that

$$
\begin{aligned}
& v \in C^{m}\left(\bar{\Omega}_{a}-\{0\}\right) \cap C^{m-1}\left(\bar{\Omega}_{a}\right), \\
& D^{\alpha} v=D^{\alpha} u \text { on } \Gamma_{a} \text { for }|\alpha| \leqq m-1
\end{aligned}
$$

and

$$
\int_{l_{h}}\left|D^{\alpha} v\right|^{2} d x_{2} \cdots d x_{n}=o\left(\exp \left(-h^{-\delta}\right)\right) \quad(h \rightarrow 0) \text { for }|\alpha| \leqq m
$$

Before proving the proposition we prepare a lemma. Let us take a function $\varphi(x) \in C^{\infty}\left(R^{1}\right)$ such that $\int_{-\infty}^{\infty} \varphi(x) d x=1$ and the carrier of $\varphi \subset\{|x| \leqq 1\}$. Set

$$
f(x, s)=\frac{1}{s} \int_{-2 s}^{2 s} \varphi((x-y) / s) d y \quad(s>0) .
$$

Then we easily see that

and

$$
f(x, s) \in C^{\infty}\left(R^{1} \times\{s>0\}\right)
$$




$$
f(x, s)= \begin{cases}1 & |x| \leqq s \\ 0 & |x|>3 s\end{cases}
$$

Further we have

\section{Lemma 2.1. There is a constant $C_{\alpha}$ such that}

$$
\left|D^{\alpha} f(x, s)\right| \leqq C_{\alpha} s^{-|\alpha|} \quad \text { in } \quad R^{1} \times\{s>0\},
$$

where $\alpha=\left(\alpha_{1}, \alpha_{2}\right)$ and $D^{\alpha}=D_{x}^{\alpha_{1}} D_{s}^{\alpha_{2}}$.

Proof. Obviously,

$$
\begin{aligned}
f(x, s) & =\int_{(x / s)-2}^{(x / s)+2} \varphi(t) d t \\
& =\int_{-2}^{2} \varphi(t) d t+\int_{0}^{x / s} \varphi(t+2) d t+\int_{x / s}^{0} \varphi(t-2) d t .
\end{aligned}
$$

Thus, if we prove the inequality (2.5) for $\int_{0}^{x / s} \varphi(t+2) d t$ and $\int_{x / s}^{0} \varphi(t-2) d t$, then we shall complete the proof.

It is easily seen that

$$
\begin{aligned}
& D_{x} \int_{0}^{x / s} \varphi(t+2) d t=\varphi\left(\frac{x}{s}+2\right) \frac{1}{s} \\
& D_{s} \int_{0}^{x / s} \varphi(t+2) d t=-\varphi\left(\frac{x}{s}+2\right) \frac{x}{s^{2}} .
\end{aligned}
$$

We have the same equality as (2.6) and (2.7) for $\int_{x / s}^{0} \varphi(t+2) d t$. Noting that $f(x, s)=0$ in $|x| \geqq 3 s$, we have proved (2.5) for $|\alpha|=1$. If we differentiate (2.6) and (2.7) any times and note that $|x / s| \leqq 3$, we obtain (2.5) for any $\alpha$.

Now we shall prove Proposition 2.1.

Proof of Proposition 2.1. Let us use the polar coordinate $\left(r, \theta_{1}, \cdots, \theta_{n-1}\right)$ with the center $\left(\frac{1}{2}, 0, \cdots, 0\right)$ such that

$$
\begin{aligned}
& x_{1}-\frac{1}{2}=r \cos \theta_{1} \\
& x_{2}=r \sin \theta_{1} \cos \theta_{2} \\
& \cdots \cdots \cdots \cdots \cdots \cdots \cdots \cdots \cdots \\
& x_{n-1}=r \sin \theta_{1} \sin \theta_{2} \cdots \sin \theta_{n-2} \cos \theta_{n-1} \\
& x_{n}=r \sin \theta_{1} \sin \theta_{2} \cdots \sin \theta_{n-2} \sin \theta_{n-1} .
\end{aligned}
$$


We denote $\left(\theta_{1}, \cdots, \theta_{n-1}\right)$ simply by $\Theta$. Set

$$
\tilde{v}(x)=\sum_{p=0}^{m-1} \frac{1}{p !}\left(r-\frac{1}{2}\right)^{p}\left(D_{r}^{p} u\right)\left(\frac{1}{2}, \Theta\right) .
$$

Then it is easily seen that

$$
D, \tilde{v}(x)=\sum_{p=l}^{n-1} \frac{1}{(p-l) !}\left(r-\frac{1}{2}\right)^{p-l}\left(D_{r}^{p} u\right)\left(\frac{1}{2}, \Theta\right)
$$

and

$$
\left(D^{l} \tilde{v}\right)\left(\frac{1}{2}, \Theta\right)=\left(D_{l}^{r} u\right)\left(\frac{1}{2}, \Theta\right) \quad \text { for } \quad l \leqq m-1 .
$$

Put $k\left(x_{1}\right)=\exp \left(-x^{-\delta-(\varepsilon / 3)}\right)$. And we set for the function $f(x, s)$ in (2.4)

$$
\zeta(x)=f\left(\sqrt{x_{1}-x_{1}^{2}}-\sqrt{\sum_{i=2}^{N} x_{i}^{2}}, k\left(x_{1}\right) / 3\right) .
$$

From now on we denote $D_{x}^{\alpha}$ simply by $D_{x}^{\prime}$ for $|\alpha|=l$. Then we see by Lemma 1.1

$$
\left|D_{x}^{l} \zeta(x)\right| \leqq c_{l}\left(x_{1} \cdots x_{n}\right)^{-c_{l}} k\left(x_{1}\right)^{-l}
$$

in a neighborhocd of the origin, where $c_{l}$ is a constant depending only on $l$. Set $v(x)=\tilde{v}(x) \zeta(x)$. Then we have

$$
D_{x}^{l} v \sim D_{x}^{l_{1}} \tilde{v} \cdot D_{x}^{l_{2}} \zeta \quad\left(l=l_{1}+l_{2}\right),
$$

where the notation $\sim$ means that the left side is a linear combination of each term on the right side. We see from (2.11)

$$
\begin{aligned}
& D_{x}^{l} v \sim D_{1_{1}^{\prime}}^{l^{\prime}} D_{\theta^{2}}^{l^{\prime \prime}} \tilde{v} \cdot D_{x^{2}}^{l_{2}} \zeta \\
& \quad\left(l_{1}^{\prime}+l_{1}^{\prime \prime}=l_{1}, l_{1}+l_{2}=l \leqq m\right) .
\end{aligned}
$$

Combining (2.8) and (2.12), we obtain

$$
\begin{gathered}
D_{x}^{l} v \sim\left(r-\frac{1}{2}\right)^{p-l_{1}^{\prime}} D_{\Theta}^{l_{1}^{\prime \prime}}\left(\left(D_{r}^{p} u\right)\left(\frac{1}{2}, \Theta\right)\right) D_{x}^{l_{2}} \zeta \\
\left(l_{1}^{\prime} \leqq p \leqq m-1\right) .
\end{gathered}
$$

We note that

$$
\left|r-\frac{1}{2}\right| \leqq k\left(x_{1}\right)
$$

in the carrier of $v$ (or $\zeta$ ).

Now let us consider each term of the right side of (2.13). If 
$l_{1}^{\prime \prime}+p \leqq m-1$, we have from (2.10), (2.14) and from the assumption on $u$,

$$
\begin{aligned}
& \left|\left(r-\frac{1}{2}\right)^{p-l_{1}^{\prime}} D_{\theta^{\prime} l^{\prime \prime}}\left(\left(D_{r}^{p} u\right)\left(\frac{1}{2}, \Theta\right)\right) D_{x^{2}}^{l_{2}} \zeta\right| \\
& \quad \leqq c\left(x_{1} \cdots x_{n}\right)^{-c} k\left(x_{1}\right)^{p-l_{1}^{\prime}-l_{2}} \exp \left(-x_{1}^{-\delta-(\varepsilon / 2)}\right) \\
& \quad \leqq c \exp \left(-x_{1}^{-\delta}\right) .
\end{aligned}
$$

If $l_{1}^{\prime \prime}+p>m-1$, we see

$$
\begin{aligned}
& \left|\left(r-\frac{1}{2}\right)^{p-l_{1}^{\prime}} D_{\theta^{\prime}}^{\prime_{1}}\left(\left(D_{r}^{p} u\right)\left(\frac{1}{2}, \Theta\right)\right) D_{x^{2}}^{l_{2}} \zeta\right| \\
& \quad \leqq c\left(x_{1} \cdots x_{n}\right)^{-c} k\left(x_{1}\right)^{p-l_{1}^{\prime}-l_{2}} \\
& \quad \leqq c\left(x_{1} \cdots x_{n}\right)^{-c} k\left(x_{1}\right)^{p+l_{1}^{\prime \prime}-l} .
\end{aligned}
$$

In particular when $l_{1}^{\prime \prime}+p>m-1$ and $l<m$, we have

$$
\text { the left side of }(2.16) \leqq c \exp \left(-x_{1}^{-\delta}\right) \text {. }
$$

Combining (2.15), (2.16) and (2.17), we have shown (2.1). Further we have obtained in the general case $l \leqq m$

$$
\left|D_{x}^{l} v\right| \leqq c\left(x_{1} \cdots x_{n}\right)^{-c} \text {. }
$$

Since the carrier of $v$ (or $\zeta$ ) is concentrated on a neighborhood of $\Gamma_{a}$, we see $\left|D_{x}^{l} v\right| \leqq c x_{1}^{-c}$. Thus we obtain

$$
\int_{t_{h}}\left|D_{x} v\right|^{2} d x_{2} \cdots d x_{n} \leqq c x_{1}^{-c} k\left(x_{1}\right) \leqq c \exp \left(-x_{1}^{-\delta}\right) .
$$

Hence we have shown (2.3). Since the equality (2.2) is trivial, we have completed the proof.

3. In this section we see how the behavior of the solutions of (1.2) or (1.4) is controled by the Cauchy data. This section is essentially based on Mizohata's result [15]. We set $\varphi_{n}\left(x_{1}\right)=\left(x_{1}+\frac{1}{n}\right)^{-n}$ and write $\varphi_{n}\left(x_{1}\right)$ simply by $\varphi$.

Lemma 3.1 (Mizohata [15]). Let $L_{1}, L_{2}$ be second order elliptic operators of the form (1.1) with sufficiently smooth coefficients in $\bar{\Omega}_{a}$. Let $w \in C^{3}\left(\bar{\Omega}_{a}\right) \cap C^{4}\left(\bar{\Omega}_{a}-\{0\}\right)$. Assume that

$$
D^{\alpha} w=0 \quad \text { on } \Gamma_{a} \quad \text { for }|\alpha| \leqq 3
$$

and 


$$
\left\|\left(D^{\alpha} w\right)(\varepsilon)\right\|^{4)} \rightarrow 0 \quad(\varepsilon \rightarrow 0) \text { for }|\alpha| \leqq 4 .
$$

Then there is a positive constant $n_{0}, h_{0}$ and $c$ independent of $n$ and $h$ such that if $n>n_{0}$ and $h<h_{0}$, then

$$
c\left\{\int_{\Omega_{h}} \varphi^{2}\left|L_{1} L_{2} w\right|^{2} d x+\tilde{c}^{2} n^{2} \varphi^{2}(h)\right\} \geqq \check{c} \sum_{|\alpha| \leqq 3} \int_{\Omega_{h}} \varphi^{2}\left|D^{\alpha} w\right|^{2} d x
$$

where $\tilde{c}=\left(h+n^{-1}\right)^{-1}$.

Proposition 3.1. Let $u$ be in $C^{4}\left(\bar{\Omega}_{a}\right) \cap C^{7}\left(\Gamma_{a}\right)$ and be a solution of (1.4) in $\Omega_{a}$. If for some $\varepsilon>0, \delta>1$,

$$
D^{\alpha} u=o\left(\exp \left(-r^{-2 \delta-\varepsilon}\right)\right) \quad(r \rightarrow 0,|\alpha| \leqq 3) \text { on } \Gamma_{a}
$$

then it holds that

$$
\int_{\Omega_{h}}\left|D^{\alpha} u\right|^{2} d x=o\left(\exp \left(-h^{-\delta}\right)\right) \quad(h \rightarrow 0,|\alpha| \leqq 3) .
$$

Proof. From Proposition 2.1 there is a function $v$ such tinat

$$
\begin{aligned}
& v \in C^{4}\left(\bar{\Omega}_{a}-\{0\}\right) \cap C^{3}\left(\bar{\Omega}_{a}\right), \\
& D^{\alpha} v=D^{a} \imath \text { on } \Gamma_{a} \text { for }|\alpha| \leqq 3
\end{aligned}
$$

and

$$
\left\|\left(D^{\alpha} v\right)(h)\right\|^{2}=o\left(\exp \left(-h^{-\delta-(\varepsilon / 3)}\right)\right) \quad(h \rightarrow 0) \text { for }|\alpha| \leqq 4 .
$$

We put $w=u-v$. Then $w$ satisfies the assumption in Lemma 3.1. Hence the inequality (3.3) holds. Here we use the following relations

$$
\left|L_{1} L_{2} w\right|^{2} \leqq 2\left(\left|L_{1} L_{2} u\right|^{2}+\left|L_{1} L_{2} v\right|^{2}\right)
$$

and

$$
\left|D^{\alpha} w\right|^{2} \geqq \frac{1}{2}\left|D^{\alpha} u\right|^{2}-\left|D^{\alpha} v\right|^{2}
$$

Since $u$ is a solution of (1.4), the inequality (3.3) becomes for sufficiently large $\tilde{c}$

$$
\begin{gathered}
c\left\{\int_{\Omega_{h}} \varphi^{2}\left|L_{1} L_{2} v\right|^{2} d x+\tilde{c} \sum_{|\alpha| \leqq 3} \int_{\Omega_{h}} \varphi^{2}\left|D^{\alpha} v\right|^{2} d x+\tilde{c}^{2} n^{2} \varphi^{2}(h)\right\} \\
\geqq \tilde{c} \sum_{|\alpha| \leqq 3} \int_{\aleph_{h}} \varphi^{2}\left|D^{\alpha} u\right|^{2} d x .
\end{gathered}
$$

4) We write $\|u(h)\|^{2}=\int_{l_{h}}|u|^{2} d x_{2} \cdots d x_{n}$. 
By (3.7) this inequality becomes

$$
\begin{gathered}
\left(\frac{h}{2}+\frac{1}{n}\right)^{2 n} c\left\{n^{2 n+1} \exp \left(-h^{-\delta-(\varepsilon / 3)}\right)+\tilde{c}^{2} n^{2}\left(\frac{1}{n}+h\right)^{-2 n}\right\} \\
\geqq \tilde{c} \sum_{|\alpha| \leqq 3} \int_{\Omega_{h / 2}}\left|D^{\alpha} u\right|^{2} d x
\end{gathered}
$$

Let us take $h+\frac{1}{n}$ sufficiently small and $n h$ sufficiently large. We easily see that in order to prove

$$
\sum_{|\alpha| \leqq 3} \int_{2_{h / 2}}\left|D^{\alpha} u\right|^{2} d x=o\left(\exp \left(-\left(\frac{h}{2}\right)^{-\delta}\right)\right) \quad(h \rightarrow 0),
$$

it is sufficient to show that we can choose $n$ in such a way that

$$
n^{2 n+1} \leqq \exp \left(\left(\frac{h}{2}\right)^{-\delta-\varepsilon_{1}}\right)
$$

and

$$
n^{4}\left(\frac{h n+2}{2 h n+2}\right)^{2 n} \leqq \exp \left(-\left(\frac{h}{2}\right)^{-\delta-\varepsilon^{\prime}}\right) \quad \varepsilon_{1}, \varepsilon^{\prime}>0
$$

where $\varepsilon_{1}$ is a given number and $\varepsilon$ will be determined later. If there is a positive number $\bar{\varepsilon}$ such that

$$
n^{1+\bar{\varepsilon}} \leqq\left(\frac{h}{2}\right)^{-\delta-\varepsilon_{1}}
$$

then (3.10) holds. Since $n h$ is sufficiently large, if we show that

$$
\left(\frac{3}{5}\right)^{2 n} \leqq \exp \left(-\left(\frac{h}{2}\right)^{-\delta-\varepsilon^{\prime}}\right)
$$

then (3.11) holds. Let us take positive numbers $\varepsilon^{\prime}, \bar{\varepsilon}$ such that

$$
\delta+\varepsilon^{\prime}<\left(\delta+\varepsilon_{1}\right) / 1+\bar{\varepsilon}_{0}
$$

Noting that $1<2 \log (5 / 3)$, we can take $n$ in such a way that

$$
\frac{1}{2 \log (5 / 3)}\left(\frac{2}{h}\right)^{\delta+\varepsilon^{\prime}}<n<\left(\frac{2}{h}\right)^{\left(\delta+\varepsilon_{1}\right) / 1+\bar{\varepsilon}} .
$$

It is easily seen that the inequality (3.14) implies (3.12), (3.13) and that $n h \rightarrow \infty$. Thus we have proved (3.9).

Secondly we consider the system of second order differential inequalities 


$$
\left|L_{p} u_{p}\right| \leqq \text { const } \sum_{q=1}^{n} \sum_{|\alpha| \leq 1}\left|D^{\alpha} u_{q}\right|, \quad p=1, \cdots, m,
$$

where each $L_{p}$ is the elliptic cperator of the form (1.1).

Proposition 3. 2. Let $u_{1}, \cdots, u_{m}$ be in $C^{2}(\bar{\Omega}) \cap C^{3}\left(\Gamma_{a}\right)$ and be solutions of the elliptic system (3.15) in $\Omega_{a}$. If for some $\varepsilon>0, \delta>1$,

$$
u_{p}, D u_{p}=o\left(\exp \left(-r^{-2 \delta-\varepsilon}\right)\right) \quad(r \rightarrow 0)
$$

on $\Gamma_{a}$ for $1 \leqq p \leqq m$, then we have

$$
\begin{aligned}
\int_{\Omega_{l}}\left|u_{p}\right|^{2} d x, \int_{\Omega_{h}}\left|D u_{p}\right|^{2} d x= & o\left(\exp \left(-h^{-5}\right)\right) \\
& (h \rightarrow 0,1 \leqq p \leqq m) .
\end{aligned}
$$

The proof of this proposition is obtained in the same way as in Proposition 3.1. Trus we shall sketch the proof briefly. From Proposition 2.1 there are functions $v_{p}(p=1, \cdots, m)$ such that

$$
\begin{aligned}
& v_{p} \in C^{2}\left(\bar{\Omega}_{a}-\{0\}\right) \cap C^{1}\left(\bar{\Omega}_{a}\right), \\
& D^{\alpha} v_{p}=D^{\alpha} u_{p} \text { on } \Gamma_{a} \text { for }|\alpha| \leqq 1
\end{aligned}
$$

and

$$
\left\|\left(D^{\alpha} v_{p}\right)(h)\right\|^{2}=o\left(\exp \left(-h^{-\delta-(\varepsilon / 3)}\right)\right) \quad(h \rightarrow 0) \text { for }|\alpha| \leqq 2 .
$$

Put $w_{p}=u_{p}-v_{p}$. Then in the same manner as in Lemma 3.1 we see that there is a positive constant $c$ independent of $n$ and $h(n, h>0)$ such that

$$
c\left\{\int_{\Omega_{h}} \varphi^{2}\left|L_{p} w_{p}\right|^{2} d x+\tilde{c}^{2} n^{2} \varphi^{2}(h)\right\} \geqq \tilde{c} \sum_{|\alpha| \leqq 1} \int_{\Omega_{h}} \varphi^{2}\left|D^{\alpha} w_{p}\right|^{2} d x,
$$

where $c=n+h^{-1}$. Then we have the inequality as in (3.8),

$$
\begin{gathered}
c \sum_{p=1}^{m}\left\{\int_{\Omega_{h}} \varphi^{2}\left|L_{p} v_{p}\right|^{2} d x+\tilde{c} \sum_{|\alpha| \leq 1} \int_{\Omega_{h}} \varphi^{2}\left|D^{\alpha} v_{p}\right|^{2} d x+\tilde{c}^{2} n^{2} \varphi^{2}(h)\right\} \\
\geqq \tilde{c} \sum_{p=1}^{m} \sum_{|\alpha| \leqq 1} \int_{\Omega_{h}} \varphi^{2}\left|D^{\alpha} u_{p}\right|^{2} d x .
\end{gathered}
$$

We proceed in the same way as in the proof of Proposition 3.1. Then we obtain (3.16).

4. In this section we shall prove the following 
Proposition 4.1. Let $u$ be in $C^{4}\left(\bar{\Omega}_{a}\right) \cap C^{7}\left(\Gamma_{a}\right)$ and be a solution of (1.4) in $\Omega_{a}$. If for some $\varepsilon>0, \delta>1$,

$$
D^{\alpha} u=o\left(\exp \left(-r^{-2 \delta-\varepsilon}\right)\right) \quad(r \rightarrow 0,|\alpha| \leqq 3) \text { on } \Gamma_{a},
$$

then, in $S_{1 / 2} \cap \Omega_{a}$, it holds that

$$
D^{\alpha} u=o\left(\exp \left(-r^{-\delta}\right)\right) \quad(r \rightarrow 0,|\alpha| \leqq 3) .
$$

Before proving the proposition we prepare a lemma. Let $x^{(0)}$ be a fized point in $R^{N}$. And let $L_{1}, L_{2}$ be second order elliptic operators with sufficiently smooth coefficients in a neighborhood of $x^{(0)}$. Further we assume that $L_{1}, L_{2}$ are real and homogeneous. Then we have

Lemma 4..$^{5)}$ Let $u$ be a $C^{4}$ function in a neighborhood of $x^{(0)}$. Then there is a constant $C$ independent of $R(R<1)$ such that

$$
\begin{aligned}
\sum_{|\alpha| \leq 3}\left|D^{\alpha} u\left(x^{(0)}\right)\right| & \leqq C R^{-p_{1}}\left\{\left(\sum_{|\alpha| \leq 3} \int_{|x-x(0)| \leq R} \mid D^{\alpha} u u_{1}^{\prime 2} d x\right)^{p_{2}}\right. \\
& \left.+\left(\int_{|x-x(0)| \leq R}\left|L_{1} L_{2} u\right|^{2} d x\right)^{p_{2}}\right\}
\end{aligned}
$$

where $p_{1}, p_{2}$ depend only on $N$.

Proof. We may assume $x^{(0)}=0$. Let us write simply by $C$ the constants independent of $R$. We take a $C^{\infty}$ function such that

$$
\phi(r)= \begin{cases}1 & \text { in } r \leqq R / 2 \\ 0 & \text { in } r \geqq R\end{cases}
$$

and $\left|D^{k} \phi\right| \leqq C R^{-k}$. Put $v=\phi u$. Then we see

$$
L_{1} L_{2} v=Q\left(D^{k} \phi \cdot D^{4-k} u\right)+\phi L_{1} L_{2} u,
$$

where $Q\left(D^{k} \phi \cdot D^{4-k} u\right)$ is a linear combination of each term $D^{k} \phi \cdot D^{4-k} u$ $(1 \leqq k \leqq 4)$.

Let $L_{i}^{(0)}(i=1,2)$ be differential operators whose coefficients are those of $L_{i}$ at $x^{(0)}$. Then there is a fundamental solution $E(x)$ of $L_{1}^{(0)} L_{2}^{(0)}$ such that

$$
\left|D^{\alpha} E(x)\right| \leqq C r^{4-N-|\alpha|}|\log r|
$$

5) From our proof it is easily seen that this lemma holds also for general fourth order elliptic operators. 
Since $D_{1}^{(0)} L_{2}^{(0)} D^{\alpha} v=D^{\alpha} L_{1} L_{2} v+D^{\alpha}\left(L_{1}^{(0)} L_{2}^{(0)}-L_{1} L_{2}\right) v$, we have

$$
D^{\alpha} u(0)=\int_{1 \leqq R} E(-x)\left\{D^{\alpha} L_{1} L_{2} v+D^{\alpha}\left(L_{1}^{(0)} L_{2}^{(0)}-L_{1} L_{2}\right) v\right\} d x
$$

When $|a| \leqq 3$, the equality (4.6) becomes

$$
\begin{gathered}
D^{\kappa} u(0)-(-1)^{|\alpha|} \int_{\sim \in \mathbb{R} R}\left(D^{x} E\right)(-x)\left\{L_{i} L_{2} v\right. \\
\left.+\left(L_{1}^{(0)} L_{2}^{(0)}-L_{1} L_{2}\right) v\right\} d x
\end{gathered}
$$

We get by (4.4) and (4.5)

$$
\begin{aligned}
& \left|\int_{r \leqq R} D^{\alpha} E(-x) L_{1} L_{2} v d x\right| \\
& \quad \leqq c R^{-4} \int_{r \leqq R} r^{-1-N}|\log r|\left(\sum_{k=0}^{3}\left|D^{k} u\right|+\left|L_{1} L_{2} u\right|\right) d x .
\end{aligned}
$$

Noting that the coefficients of $L_{1}^{(0)} L_{2}^{(0)}-L_{1} L_{2}$ have order $O(\gamma) \quad(\gamma \rightarrow 0)$, we have from (4.5) by the integration by parts

$$
\begin{aligned}
& \left|\int_{r \leqq R} D^{\alpha} \mathbb{E}(-x)\left(L_{1}^{(0)} L_{2}^{(0)}-L_{1} L_{2}\right) v d x\right| \\
& \quad \leqq C \int_{r \leqq R} r^{1-N}|\log r|\left|D^{3} v\right| d x \\
& \quad \leqq C R^{-3} \int_{r \leqq R} r^{1-N}|\log r|\left(\sum_{k=0}^{3}\left|D^{k} u\right|\right) d x .
\end{aligned}
$$

Combining (4.7), (4.8) and (4.9), we obtain

(4.10) $\left|D^{\alpha} u(0)\right| \leqq C R^{-4} \int_{r \leqq R} r^{1-N}|\log r|\left(\sum_{k=0}^{3}\left|D^{k} u\right|+\left|L_{1} L_{2} u\right|\right) d x$.

Put $m=\max _{r \leqq R}\left(\sum_{k=0}^{3}\left|D^{k} u\right|\right)$. Then by Hölder's inequality we have

$$
\begin{aligned}
& \int_{r \leqq R} r^{1-N}|\log r| m^{-1}\left(\sum_{k=0}^{3}\left|D^{k} u\right|\right) d x \\
& \quad \leqq\left(\int_{r \leqq R}\left(r^{1-N}|\log r|\right)^{p} d x\right)^{1 / p}\left(\int_{1 \leqq R}\left(m^{-1} \sum_{k=0}^{3}\left|D^{k} u\right|\right)^{q} d x\right)^{1 / q},
\end{aligned}
$$

where $p^{-1}+q^{-1}=1$. We can take $p, q$ in such a way that $q>2$ and the right side of (‥11) is finite. Since

$$
\left(m^{-1} \sum_{k=0}^{3}\left|D^{k} u\right|\right)^{a} \leqq\left(m^{-1} \sum_{k=0}^{3}\left|D^{k} u\right|\right)^{2}
$$

the left side of (4.10) is estimated from above by 


$$
C R^{-p_{1}}\left(\int_{r \leqq R}\left(\sum_{k=0}^{3}\left|D^{k} u\right|^{2}\right) d x\right)^{p_{2}}
$$

Similarly we have

$$
\int_{r \leqq R} r^{1-N}|\log r|\left|L_{1} L_{2} u\right| d x \leqq C R^{-p_{1}}\left(\int_{r \leqq R}\left|L_{1} L_{2} u\right|^{2} d x\right)^{p_{2}}
$$

Thus from (4.10) we have completed the proof.

Now we shall prove Proposition 4.1.

Proof of Proposition 4. 1. For the point $x^{(0)}$ in $S_{1 / 2}$ we denote by $r_{1}\left(x^{(0)}\right)$ the radius of a sphere tangent to $S_{1}$ whose center is $x^{(0)}$. It is easily seen that

$$
r_{1}\left(x^{(0)}\right) \sim x_{1}^{(0)} \quad\left(x_{1}^{(0)} \rightarrow 0\right) .
$$

By Proposition 3.1 we have

$$
\begin{array}{r}
\int_{\Omega_{r_{1}\left(x^{(0)}\right)+x_{1}^{(0)}}} \mid D^{\alpha} u_{1}^{2} d x=o\left(\exp \left(-x_{1}^{(0)-\delta-(\varepsilon / 3)}\right)\right) \\
\left(x_{1}^{(0)} \rightarrow 0,|\alpha| \leqq 3\right) .
\end{array}
$$

Let us apply Lemma 4.1 to the sphere with the center $x^{(0)}$. Then we obtain from (4.3) and (4.12)

$$
D^{\alpha} u\left(x_{0}\right)=o\left(\exp \left(-x_{1}^{(0)-\delta}\right)\right) \quad\left(x_{1}^{(0)} \rightarrow 0,|\alpha| \leqq 3\right) .
$$

Thus we have completed the proof.

We note that Lemma 4.1 holds also for second order elliptic operators. Hence by Proposition 3. 2 we have the following

Proposition 4.2. Let $u_{p}(p=1, \cdots, m)$ be in $C^{2}\left(\bar{\Omega}_{a}\right) \cap C^{3}\left(\Gamma_{a}\right)$ and be solutions of (1.2) in $\Omega_{a}$. Then, if for some $\varepsilon>0, \delta>1$,

$$
D^{\alpha} u_{p}=o\left(\exp \left(-r^{-2 \delta-\varepsilon}\right)\right) \quad(r \rightarrow 0,|\alpha| \leqq 1, p \leqq m)
$$

on $\Gamma_{a}$, then in $S_{1 / 2} \cap \Omega_{a}$

$$
D^{\alpha} u_{p}=o\left(\exp \left(-r^{-\delta}\right)\right) \quad(r \rightarrow 0,|\alpha| \leqq 1, p \leqq m) .
$$

5. We prepare an energy estimate of Carleman's type for second order elliptic operators. We proceed along the method developed by Pederson [17], Protter [18] and Shirota [19]. 
Definition. If a function $v(x)$ satisfies the following conditions, we say that $v$ belongs to $\mathscr{F}_{m, \delta}\left(S_{d}\right)$ :

(i) $v \in C^{m-1}\left(R^{N}\right) \cap C^{m}\left(\overline{S_{d}}-\{0\}\right)$ and the carrier of $v$ is contained in $\overline{S_{d}}$.

(ii) For some $\varepsilon>0$,

$$
D^{\alpha} v=o\left(\exp \left(-r^{-s-\varepsilon}\right)\right) \quad(r \rightarrow 0,|\alpha|<m) \text { in } \bar{S}_{d} .
$$

Now we set $\Phi(x)=x_{1} / r^{2}$. Then an easy computation shows that

$$
\begin{aligned}
& \Phi_{x_{1}}=\frac{r^{2}-2 x_{1}^{2}}{r_{4}}, \quad \Phi_{x_{i}}=-2 x_{1} x_{i} / r^{4} \quad(i \neq 1) \\
& \Phi_{x_{1} x_{1}}=\left(-2 x_{1}\right)\left(\frac{3}{r^{4}}-\frac{4 x_{1}^{2}}{r^{6}}\right) \\
& \Phi_{x_{i} x_{i}}=\left(-2 x_{1}\right)\left(\frac{1}{r^{4}}-\frac{4 x_{i}^{2}}{r^{6}}\right) \quad(i \neq 1)
\end{aligned}
$$

and

$$
\left|D^{k} \Phi\right| \leqq c r^{-k-1} \quad(0 \leqq k \leqq 2) .
$$

Let us note that the condition (5.1) implies

$$
D_{x}^{\alpha} v=o\left(\exp \left(-\Phi^{\delta+\varepsilon}\right)\right) \quad(r \rightarrow 0,|\alpha|<m) \text { in } \overline{S_{d}} .
$$

Let $L$ be a second order homogeneous elliptic operator of the form (1.1) defined in a neighborhood of the origin. And we assume $L=\Delta$ at the origin. Then we have

Proposition 5.1. There are positive constants $d_{0}$, $n_{0}$ depending only on $N$ and $a_{i j}$ such that if $0<d \leqq d_{0}, n \geqq n_{0}$, and $\delta>N-1$ it holds for any $v \in \mathscr{F}_{2, \delta}\left(S_{d}\right)$

$$
\begin{aligned}
& c \int r^{4} \Phi^{1-\delta} \exp \left(2 n \Phi^{\delta}\right)(L v)^{2} d x \\
& \quad \geqq n^{3} \int r^{-4}{\Phi^{2 \delta-3}}^{2} \exp \left(2 n \Phi^{\delta}\right) v^{2} d x+n \int \Phi^{-1} \exp \left(2 n \Phi^{\delta}\right)|\nabla v|^{2} d x,
\end{aligned}
$$

where $c$ is a constant depending on $d_{0}$ and $n_{0}$ but independent of $n$.

Proof. We can write

$$
a_{i j}=\delta_{i j}+b_{i j}, \quad\left|b_{i j}\right| \leqq c r .
$$


Let us denote simply by $c$ the positive constant independent of $n$ and d. We see by (5.2)

$$
|\nabla \Phi|^{2}=r^{-4}
$$

Let us put $g=\exp \left(-n \Phi^{\delta}\right)$ and $v=z g$. It is easily seen that $z \in C^{1}\left(\overline{S_{d}}\right)$ $\cap C^{2}\left(\overline{S_{d}}-\{0\}\right)$ and the carrier of $z \subset \bar{S}_{d}$. Further we note that if $k+l \leqq 3$, and $k \neq 3, i \neq 3$,

$$
D_{x}^{k} z D_{x}^{l} z=o\left(\exp \left(-\Phi^{\varepsilon / 2}\right)\right) \quad(r \rightarrow 0) \text { in } \overline{S_{d}} .
$$

Obviously

$$
g^{-1} L v=L z-2 n \delta \phi^{\delta-1}\left(\sum a_{i j} z_{x_{i}} \phi_{x_{j}}\right)+z g^{-1} L g
$$

We use the following inequality for the right of (5.9)

$$
(X+Y+Z)^{2} \geqq 2 Y(X+Z) .
$$

Then

$$
\begin{aligned}
g^{-2}(L v)^{2} \geqq & -4 n \delta \Phi^{\delta-1}\left(\sum a_{i j} z_{x_{i}} \Phi_{x_{j}}\right) L z \\
& -4 n \delta \Phi^{\delta-1}\left(\sum a_{i j} z_{x_{i}} \Phi_{x_{j}}\right) z g^{-1} L g .
\end{aligned}
$$

Thus we have

$$
\begin{aligned}
\int r^{4} \Phi^{1-\delta} g^{-2}(L v)^{2} d x \geqq & -4 n \delta \int r^{4} L z\left(\sum_{i, j} a_{i j} z_{x_{i}} \Phi_{x_{j}}^{5}\right) d x \\
& -4 n \delta \int r^{4}\left(\sum_{i, j} a_{i j} z_{i_{i}} \Phi_{x_{j}}\right) z g^{-1} \operatorname{Lg} d x,
\end{aligned}
$$

where the integral domain is $S_{d}$. We write

$$
\begin{aligned}
& -4 n \delta r^{1} L z\left(\sum a_{i j} z_{x_{i}} \Phi_{x_{j}}\right) \\
& =-4 n \delta r^{4}\left(\sum_{i}\left(1+b_{i i}\right) z_{x_{i} x_{i}}+\sum_{i \neq j} a_{i j} z_{x_{i} x_{j}}\right) \\
& \quad \times\left(\sum_{i}\left(1+b_{i i}\right) z_{x_{i}} \Phi_{x_{i}}+\sum_{i \neq j} a_{l j} z_{x_{i}} \Phi_{x_{j}}\right) \\
& =-4 n \delta r^{ \pm}(\Delta z)(\nabla z \cdot \nabla \Phi)-4 n \delta R,
\end{aligned}
$$

where $R$ is the sum of the remained terms.

In general for any function $f(x)$ it holds

$$
\int f z_{x_{i} x_{i}} z_{x_{j}} d x=-\int f_{x_{i}} z_{y_{i}} z_{x_{j}} d x+\frac{1}{2} \int f_{x_{j}} z_{x_{i}}^{2} d x
$$

Further we have 
(5. 14) $\int f z_{x_{i} x_{i}} z_{x_{k}} d x=\frac{1}{2}\left(-\int f_{x_{j}} z_{y_{i}} z_{x_{k}} d x+\int f_{x_{k}} z_{x_{i}} z_{z_{j}} d x-\int f_{x_{i}} z_{x_{k}} z_{x_{j}} d x\right)$

In fact

$$
\begin{aligned}
& \int f z_{x_{i} \lambda} z_{x_{k}} d x=-\int f_{x_{j}} z_{x_{i}} z_{x_{k}} d x-\int f z_{x_{i} z_{\lambda_{k} x}} d x,
\end{aligned}
$$

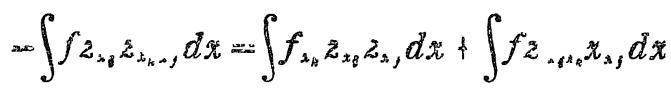

and

$$
\int f z_{x_{i} x_{k}} z_{x_{j}} d x=-\int f_{\lambda_{i}} z_{x_{k}} z_{\lambda_{j}} d x-\int f z_{z_{k}} z_{x_{j} x_{i}} d x
$$

Combining (5.15), (5.16) and (5.17), we obtain (5.14).

Applying (5.13) and (5.14) to each of $R$, we see from (5.12)

$$
\left.\left|\int R d x \leqq r d \int \Phi^{-1}\right| \Delta z\right|^{2} d x
$$

Now let us show the following inequality

$$
\int r^{4}(\Delta z)(\nabla z \cdot \nabla \Phi) d x \leqq 0
$$

We get from (5.7)

$$
r^{4}(\nabla z \cdot \nabla \Phi)=z_{x_{1}}\left(r^{2}-2 x_{1}^{2}\right)-2 x_{1} \sum_{i=2}^{N^{r}} z_{x_{i}} x_{i}
$$

Thus

$$
\begin{aligned}
& \int r^{4}(\Delta z)(\nabla z \cdot \nabla \Phi) d x \\
= & \int z_{x_{1} x_{1}} z_{x_{1}}\left(r^{2}-2 x_{1}^{2}\right) d x+\sum_{i=2}^{N} \int z_{x_{i} x_{i}} z_{x_{1}}\left(r^{2}-2 x_{1}^{2}\right) a^{7} x \\
& \quad-\int z_{x_{1} x_{1}} 2 x_{1}\left(\sum_{i=2}^{N} z_{x_{i} x_{i}}\right) d x-\sum_{i=2}^{N} \int z_{x_{i} x_{i}} 2 x_{1}\left(\sum_{j=2}^{N} z_{x_{j}} x_{j}\right) d x \\
= & J_{1}+J_{2}-J_{3}-J_{4} .
\end{aligned}
$$

By (5.8) we can integrate by parts each $J_{i}$. Integrating by parts, we can verify

$$
\begin{aligned}
& J_{1}=\int z_{x_{1}}^{2} x_{1} d x \\
& J_{2}=-\int\left(\sum_{i=2}^{N} z_{x_{i}}^{2}\right) x_{1} d x-\int\left(\sum_{i=2}^{N} 2 x \cdot z_{x_{i}}\right) z_{x_{1}} d x
\end{aligned}
$$




$$
J_{3}=(N-1) \int z_{x_{1}}^{2} x_{1} d x-\int\left(\sum_{i=2}^{N} 2 x_{i} z_{x_{i}}\right) z_{x_{1}} d x
$$

and

$$
J_{4}=\sum_{i=2}^{N} \int(N-2) z_{x_{i}}^{2} x_{1} d x
$$

Combining these integrals, we get

$$
\int r^{4}(\Delta z)(\nabla z \cdot \nabla \Phi) d x=(2-N) \sum_{i=1}^{N} \int z_{x_{1}}^{2} x_{1} d x \leqq 0
$$

Hence the inequality (5.19) has been shown. We have from (5.12), (5.18) and (5.19)

$$
\int r^{4} L z\left(\sum_{i, j} a_{i j} z_{x_{i}} \Phi_{x_{j}}\right) \leqq c d \int \Phi^{-1}|\nabla z|^{2} d x
$$

Now we estimate the last integral in (5.11). A computation shows

$$
g^{-1} L g=\sum_{k, l} a_{k l}\left\{\left(n^{2} \delta^{2} \Phi^{2(\delta-1)}-n \delta(\delta-1) \Phi^{\delta-2}\right) \Phi_{x_{k}} \Phi_{x_{l}}-n \delta \Phi^{\delta-1} \Phi_{x_{k} x_{l}}\right\}
$$

Thus

$$
\begin{aligned}
& -4 n \delta \int r^{4}\left(\sum_{i, j} a_{i j} z_{x_{i}} \Phi_{x_{j}}\right) z g^{-1} \operatorname{Lg} d x \\
= & 2 n \delta \sum_{i, j, k, l} \int\left(r^{4} a_{i j} \Phi_{x_{j}} n^{2} \delta^{2} a_{k l} \Phi^{2(\delta-1)} \Phi_{x_{k}} \Phi_{x_{l}}\right)_{x_{i}} z^{2} d x \\
& -2 n \delta \sum_{i, j, k, l} \int\left(r^{4} a_{i j} \Phi_{x_{j}} n \delta(\delta-1) a_{k l} \Phi^{\delta-2} \Phi_{x_{k}} \Phi_{x_{l}}\right)_{x} z^{2} d x \\
& -2 n \delta \sum_{i, j, k_{, l}} \int\left(r^{4} a_{i j} \Phi_{x_{j}} n \delta a_{k l} \Phi^{\delta-1} \Phi_{x_{k} x_{l}}\right)_{x_{i}} z^{2} d x .
\end{aligned}
$$

The first integral on the right of (5.21) contains the following three terms:

$$
\begin{aligned}
& M_{1}=4 n^{3} \delta^{3}(\delta-1) \sum_{i, j, k, l} \int r^{4} a_{i j} a_{k l} \Phi_{x_{i}} \Phi_{x_{j}} \Phi_{x_{k}} \Phi_{x_{l}} \Phi^{2 \delta-3} z^{2} d x, \\
& M_{2}=2 n^{3} \delta^{3} \sum_{i, j, k, l} \int r^{4} a_{i j} a_{k l} \Phi_{x_{j} x} \Phi_{x_{k}} \Phi_{x_{l}} \Phi^{2(\delta-1)} z^{2} d x
\end{aligned}
$$

and

$$
M_{3}=2 n^{3} \delta^{3} \sum_{i, j, k, l} \int r^{4} a_{i j} a_{k l} \Phi_{x_{j}}\left(\Phi_{x_{k}} \Phi_{x_{l}}\right)_{x_{t}} \Phi^{2(\delta-1)} z^{2} d x
$$

From the positive definiteness of $L$ and (5.7) we have 


$$
M_{1} \geqq c n^{3} \delta^{3}(\delta-1) \int r^{-4} \Phi^{2 \delta-3} z^{2} d x
$$

We decompose the integral $M_{2}$ into

$$
\begin{aligned}
M_{2} & =2 n^{3} \delta^{3} \sum_{i, j, k, l} \int r^{4} \delta_{i j} \delta_{k} \Phi_{x_{i} x} \Phi_{x_{k}} \Phi_{x_{l}} \Phi^{2(\delta-1)} z^{2} d x \\
& +2 n^{3} \delta^{3} \sum_{i, j, k, l} \int r^{4}\left(a_{i j} a_{k}-\delta_{i j} \delta_{k l}\right) \Phi_{x_{i} x} \Phi_{x_{k}} \Phi_{x_{l}} \Phi^{2(\delta-1)} z^{2} d x
\end{aligned}
$$

Since we have assumed $a_{\imath j}=\delta_{i j}$ at the origin, the second integral of (5.23) is absorbed by the right of (5.22) if $d \leqq d_{0}$ for sufficiently small $d_{0}$. The first integral of $(5.23)$ becomes

$$
2 n^{3} \delta^{3} \int r^{4} \Delta \Phi(\nabla \Phi)^{2} \Phi^{2(\delta-1)} z^{2} d x
$$

Let us note that $\Delta \Phi=2(2-N) x_{1} / r^{4}$ by (5.2). Then we see from (5. 7)

$$
\text { the first integral of (5.23) }
$$

$$
=4(2-N) n^{3} \delta^{3} \int x_{1} r^{-4} \Phi^{2(\xi-1)} z^{2} d x \text {. }
$$

Since the constant $c$ on the right of (5.22) can be taken as arbitrarily near to 4 for sufficiently small $d_{0}$, the integral (5.24) is absorbed by the right of (5.22) for $\delta>N-1$. Thus we see that the term $M_{2}$ is absorbed by $M_{1}$.

Secondly we shall show that the term $M_{3}$ is absorbed by the right of (5.22). Let us decompose $M_{3}$ in a similar way to (5.23). We see

$$
\begin{aligned}
& \sum_{i, j, k, l} \delta_{i j} \delta_{k l} \Phi_{x_{j}}\left(\Phi_{\lambda_{k}} \Phi_{\imath_{l}}\right)_{\lambda_{i}} \\
= & \sum_{i, k} \bar{\emptyset}_{x_{i}}\left(\Phi_{x_{k}}^{2}\right)_{x_{i}}=\sum_{i} \Phi_{x_{i}}\left(r^{-4}\right)_{\lambda_{i}}=-4 \sum_{i} r^{-6} x_{i} \Phi_{x_{i}} .
\end{aligned}
$$

On the other hand we have from (5.2)

$$
\sum_{i} x_{i} \Phi_{x_{i}}=-x_{1} r^{-2}
$$

Thus we get

$$
\sum_{i, j, k, l} \delta_{i j} \delta_{k l} \Phi_{x_{j}}\left(\Phi_{x_{k}} \Phi_{x_{l}}\right)_{x_{\imath}} \geqq 0 .
$$

Hence the term $M_{3}$ is absorbed by the right of (5.22). More easily 
we can see that the remaining terms on the right of (5.21) are absorbed by the right of (5.22) if we take $n \geq n_{0}$. Therefore combining $(5.11),(5.20),(5.21)$ and $(5.22)$, we obtain

$$
\begin{aligned}
& n^{3} \int r^{-4} \Phi^{2 \delta-3} \exp \left(2 n \Phi^{\delta}\right) v^{2} d x \\
& \leqq c\left(\int r^{4} \Phi^{1-8} \exp \left(2 n \Phi^{\delta}\right)(\Sigma v)^{2} d x\right. \\
& \left.\quad+n d \int \Phi^{-1} \exp \left(2 n \Phi^{\delta}\right)|\nabla v|^{2} d x\right) .
\end{aligned}
$$

Let us put $q=\Phi^{-1} \exp \left(2 n \Phi^{\delta}\right)$. Then

$$
q_{i_{s}}^{2} \leqq c n^{2} r^{-4} \Phi^{2 \delta-4} \exp \left(4 n \Phi^{S}\right)
$$

And we see

$$
\int q v L v d x=-\int q a_{i j} v_{x_{i}} v_{x_{j}} d x-\int\left(q a_{i j}\right)_{x_{j}} v v_{x_{i}} d x
$$

Hence we have

$$
\int q(\nabla v)^{2} d x \leqq\left|\int\left(q a_{i j}\right)_{x_{j}} v v_{x_{i}} d x\right|+\left|\int q v \operatorname{Lv} d x\right|
$$

By Cauchy's inequality we get from (5.26)

$$
\begin{aligned}
& \left|\left(q a_{i j}\right)_{x_{j}} v v_{x_{i}}\right|=\left|\Phi^{-1 / 2} \exp \left(n \Phi^{\delta}\right) v_{x_{i}}\right| \cdot\left|\Phi^{1 / 2} \exp \left(-n \Phi^{\delta}\right)\left(q a_{i_{j}}\right)_{x_{j}} v\right| \\
& \leqq c\left\{\varepsilon \Phi^{-1} \exp \left(2 n \Phi^{\delta}\right)|\nabla v|^{2}+\varepsilon^{-1} n^{2} \gamma^{-4} \Phi^{2 \delta-3} \exp \left(2 n \Phi^{\delta}\right) v^{2}\right\} .
\end{aligned}
$$

Here we take $\varepsilon$ as sufficiently small. On the other hand we have

$$
\begin{aligned}
& 2|q v L v| \leqq 2 \sqrt{2 r^{-4} \Phi^{\delta-2}} \cdot \sqrt{n^{-1} \gamma^{4} \Phi^{1-\delta}} \exp \left(2 n \Phi^{\delta}\right)|v L v| \\
& \leqq n r^{-4} \Phi^{\delta-\Omega} \exp \left(2 n \Phi^{\delta}\right) v^{2}+n^{-1} r^{4} \Phi^{1-\delta} \exp \left(2 n \phi^{\delta}\right)(L v)^{2} .
\end{aligned}
$$

Combining (5.27), (5.28) and (5.29), we have

$$
\begin{aligned}
& \int \Phi^{-1} \exp \left(2 n \Phi^{\delta}\right)(\nabla v)^{2} d x \\
& \leqq c\left\{n^{2} \int r^{-1} \Phi^{2 \delta-3} \exp \left(2 n \Phi^{\delta}\right) v^{2} d x\right. \\
& \left.\quad+n^{-1} \int r^{+} \Phi^{1-\delta} \exp \left(2 n \Phi^{\delta}\right)(L v)^{2} d x\right\} .
\end{aligned}
$$

Substituting this inequality into (5.25), we obtain 


$$
\begin{gathered}
n^{3}(1-c d) \int r^{-4} \Phi^{2 \delta-3} \exp \left(2 n \Phi^{\delta}\right) v^{2} d x \\
\leqq c \int r^{4} \Phi^{1-\delta} \exp \left(2 n \Phi^{\delta}\right)(L v)^{2} d x
\end{gathered}
$$

where $d_{0}$ is taken as more sufficiently small. By (5.30) and (5.31) we complete the proof.

Now let $\gamma_{1}, \cdots, \gamma_{N}$ be the fixed real numbers such that $\sum_{i=1}^{N} r_{i}^{2}=1$. Then we put for $d>0$

$$
\widetilde{S}_{d}=\left\{r^{2}<d\left(\gamma_{1} x_{1}+\cdots+\gamma_{N} x_{N}\right)\right\} .
$$

The set $\widetilde{S}_{d}$ is an open sphere with the center $\left(\frac{d}{2} \gamma_{1}, \cdots, \frac{d}{2} \gamma_{N}\right)$ and the radius $d / 2$. Further we set $\widetilde{\Phi}(x)=\sum_{i=1}^{N} \gamma_{i} x_{i} / r^{2}$.

Definition. If a function $v(x)$ satisfies the following conditions, we say that $v$ belongs to $\mathscr{I}_{2, d}\left(\widetilde{S}_{d}\right)$ :

(i) $v \in C^{1}\left(R^{N}\right) \cap C^{2}\left(\overline{\widetilde{S}_{d}}-\{0\}\right)$ and the carrier of $v$ is contained in $\overline{\widetilde{S_{d}}}$.

(ii) For some $\varepsilon>0$

$$
v, D_{x} v=o\left(\exp \left(-r^{-\delta-\varepsilon}\right)\right) \quad(r \rightarrow 0) \text { in }{\widetilde{S_{d}}}_{d}
$$

Now we also assume that $L=\Delta$ at the origin as in Proposition 5. 1. Then we have by an adequate orthogonal transformation for (5.5).

Corollary 5.1. There are positive constants $d_{0}, n_{0}$ depending only on $a_{i j}$ such that if $0<d \leqq a_{0}^{\prime}, n \geqq n_{0}$ and $\delta>N-1$, it holds for any $v \in \mathscr{F}_{2, \delta}\left(\widetilde{S_{d}}\right)$

$$
\begin{aligned}
& c \int r^{4} \widetilde{\Phi}^{1-\delta} \exp \left(2 n \widetilde{\Phi}^{\delta}\right)(L v)^{2} d x \\
& \quad \geqq n^{3} \int r^{-4} \widetilde{\Phi}^{2 \delta-3} \exp \left(2 n \tilde{\Phi}^{\delta}\right) v^{2} d x+n \int \widetilde{\Phi}^{-1} \exp \left(2 n \widetilde{\Phi}^{\delta}\right)|\nabla v|^{2} d x
\end{aligned}
$$

where $c$ is the same constant as in Proposition 5.1.

We have assumed that $L=\Delta$ at the origin in Proposition 5. 1 and Corollary 5.1. If we eliminate this assumption, the statement of Proposition 5. 1 holds also, that is, we can prove

Proposition 5.2. There are positive constants $d_{0}, n_{0}$ and $o_{0}$ 
depending only on $a_{i j}$ such that if $0<d \leqq d_{0}, n \geqq n_{0}$ and $\delta>\delta_{0}$, it holds for any $v \in \mathscr{F}_{2, \delta}\left(S_{d}\right)$

$$
\begin{aligned}
& c \int r^{4} \Phi^{1-\delta} \exp \left(2 n \Phi^{\delta}\right)(L v)^{2} d x \\
& \quad \geqq n^{3} \int r^{-4} \Phi^{2 \delta-3} \exp \left(2 n \Phi^{\delta}\right) v^{2} d x+n \int \Phi^{-1} \exp \left(2 n \Phi^{\delta}\right)|\nabla v|^{2} d x,
\end{aligned}
$$

where $c$ is a constant depending on $d_{0}$ and $n_{0}$ but independent of $n$.

Proof. Let $L^{(0)}$ te the operator whcse coefficients are those of $L$ at the origin. The operator $L_{N}^{(0)}$ is reduced to $\sum_{i=1}^{N} \lambda_{i} D_{\lambda_{i}^{\prime}}$ by an orthogonal transformation $x^{\prime}=T x\left(x_{i}=\sum_{j=1}^{N} s_{i j} x_{j}^{\prime}\right)$. Secondly the operator $\sum_{i=1}^{N} \lambda_{i} D_{x_{i}^{\prime}}^{2}$ is reduced to $\Delta$ by the trarsformation $x^{\prime \prime}=\Lambda x^{\prime}\left(x_{i}^{\prime}=\lambda_{i i} x^{\prime \prime}\right)$. On the other hand the sphere $S_{d}$ is mapped into the following set:

$$
\sum_{i=1}^{N}\left(\sum_{j=1}^{N} \lambda_{j} s_{\iota j} x_{j}^{\prime \prime}\right)^{2}<d \sum_{j=1}^{N} \lambda_{j} s_{1 j} x_{j}^{\prime \prime}
$$

Obviously there is a positive constant $c$ such that

$$
c^{-1} \sum_{i=1}^{N} x_{i}^{\prime \prime 2} \leqq \sum_{i=1}^{N}\left(\sum_{j=1}^{N} \lambda_{j} s_{i j} x_{j}^{\prime \prime}\right)^{2} \leqq c \sum_{i=1}^{N} x_{i}^{\prime \prime 2}
$$

We put

$$
\gamma=\sqrt{\sum_{j=1}^{N}\left(\lambda_{j} s_{1 j}\right)^{2}}, \gamma_{j}=\gamma^{-1} \lambda_{j} s_{1 j} \quad(j=1, \cdots, N) \text {. }
$$

Setting $\widetilde{\Phi}=\sum_{j=1}^{N} r_{j} x_{j}^{\prime \prime} / \sum_{j=1}^{N} x_{j}^{\prime \prime 2}$, we have the inequality (5.32) for any $v\left(x^{\prime \prime}\right) \in \mathscr{F}_{2, \delta}\left({\widetilde{S_{d}}}\right)\left(0<d \leqq d_{0}\right)$ from Corollary 5.1 , that is

$$
\begin{aligned}
& c \int \tilde{r}^{4} \tilde{\Phi}^{1-\delta} \exp \left(2 n \tilde{\Phi}^{\delta}\right)\left(L^{\prime \prime} v\right)^{2} d x^{\prime \prime} \\
& \quad \geqq n^{3} \int \tilde{r}^{-4} \tilde{\Phi}^{2 \delta-3} \exp \left(2 n \tilde{\Phi}^{\delta}\right) v^{2} d x^{\prime \prime}+n \int \tilde{\Phi}^{-1} \exp \left(2 n \tilde{\Phi}^{\delta}\right)|\nabla v|^{2} d x^{\prime \prime},
\end{aligned}
$$

where $\tilde{r}=\left(\sum_{i=1}^{N} x_{i}^{\prime 2}\right)$ and $L^{\prime \prime}$ is the transformed operator of $L$ by $x^{\prime \prime}=\Lambda T x$.

Let us note that if (5.34) holds, we have by (5.35)

$$
\sum_{i=1}^{N} x_{i}^{\prime \prime 2}<d c \gamma \sum_{j=1}^{N} r_{j} x_{j}^{\prime \prime}
$$

Thus taking $d_{0}^{\prime}$ sufficiently small, we see that if $v(x) \in \mathscr{F}_{2, \delta}\left(S_{d}\right)$ $\left(0<d \leqq d_{0}^{\prime}\right)$, then $v\left(x^{\prime \prime}\right) \in \mathscr{F}_{2, \delta}\left({\widetilde{S_{d}}}\right)\left(0<d \leqq d_{0}\right)$. Hence the inequality 
(5. 36) holds for the function $v\left(x^{\prime \prime}\right)$ of $v(x) \in \mathscr{F}_{2, \delta}\left(S_{d}\right) \quad\left(0<d \leqq d_{0}^{\prime}\right)$.

Now from (5.35) we easily see

$$
\frac{r^{-1}\left(\sum_{j} \lambda_{j} s_{1 j} x_{j}^{\prime \prime}\right)}{c \sum_{i}\left(\sum_{j} \lambda_{j} s_{i j} x_{j}^{\prime \prime}\right)^{2}} \leqq \widetilde{\Phi} \leqq \frac{r^{-1}\left(\sum_{j} \lambda_{j} s_{1 j} x_{j}^{\prime \prime}\right)}{c^{-1} \sum_{i}\left(\sum_{j} \lambda_{j} s_{i j} x_{j}\right)^{2}},
$$

that is,

$$
\frac{r^{-1}}{c} \frac{x_{1}}{r_{2}} \leqq \widetilde{\Phi} \leqq \frac{r^{-1}}{c^{-1}} \frac{x_{1}}{r^{2}}
$$

And we see for another positive constant $c$

$$
c^{-1} \sum_{i=1}^{N} x_{i}^{2} \leqq \sum_{i=}^{N} x_{i}^{\prime \prime 2} \leqq c \sum_{i=1}^{N} x_{i}^{2}
$$

Performing the inverse transformation $x=T^{-1} \Lambda^{-1} x^{\prime \prime}$ on the inequality (5.36), we apply the relations (5.37) and (5.38). Then we obtain (5.33) for any $v(x) \in \mathscr{F}_{2, \delta}\left(S_{d}\right)\left(0<d \leqq d_{0}^{\prime}\right)$.

6. We prove the similar estimate to that in the previous section for fourth order elliptic operators. Let $L_{1}$ and $L_{2}$ be second order homogeneous operators of the form (1.1) with sufficiently smooth coefficients in a neighborhood of the origin. Then we have

Proposition 6.1. There are positive constants $d_{0}$ and $n_{0}$ depending only on $L_{1}$ and $L_{2}$ such that if $0<d<d_{0}, n>n_{0}$ and $\delta>\delta_{0}$, it holds for any $v \in \mathscr{F}_{4, \delta}\left(S_{d}\right)$ that

$$
\begin{aligned}
& c \int r^{12} \Phi^{5-4 \delta} \exp \left(2 n \Phi^{\delta}\right)\left(L_{1} L_{2} v\right)^{2} d x \\
& \geqq \int r^{12} \Phi^{5-4 \delta} \exp \left(2 n \Phi^{\delta}\right)\left(\sum_{|\alpha|=3}\left|D^{\alpha} v\right|^{2}\right) d x \\
& \quad+n^{2} \int r^{8} \Phi^{3-2 \delta} \exp \left(2 n \Phi^{\delta}\right)\left(\sum_{|\alpha|=2}\left|D^{\alpha} v\right|^{2}\right) d x \\
& \quad+n^{4} \int \Phi^{-1} \exp \left(2 n \Phi^{\delta}\right)(\nabla v)^{2} d x \\
& \quad+n^{6} \int r^{-4} \Phi^{2 \delta-3} \exp \left(2 n \Phi^{\delta}\right) v^{2} d x .
\end{aligned}
$$

Proof. We easily see 


$$
\int q v \Delta v d x=-\int q(\nabla v)^{2} d x+\frac{1}{2} \int(\Delta q) v^{2} d x
$$

and

$$
\int q v_{x_{i} x_{i}} v_{x_{j} x_{j}} d x=\int q v_{x_{i} x_{j}}^{2} d x-\frac{1}{2} \int\left(q_{x_{i} x_{i}} v_{x_{j}}^{2}-2 q_{x_{i} x_{j}} v_{x_{i}} v_{x_{j}}+q_{x_{j} x_{j}} v_{x_{i}}^{2}\right) d x
$$

Now we consider the integral

$$
\int q(\Delta v)^{2} d x=\int\left(q \sum_{i=1}^{N} v_{x_{i} x_{i}}^{2}+q \sum_{i \neq j} v_{x_{i} x_{i}} v_{x_{j} x_{j}}\right) d x .
$$

Let us substitute (6.3) into the second term on the right. Then we have

$$
\int q\left(\sum_{i, j} v_{x_{x} x_{j}}^{2}\right) d x=\int q(\Delta v)^{2} d x+\int F\left(q_{x_{i} x_{j}}, v_{x_{k}}\right) d x
$$

where $F$ is a sum of products of $v_{x_{k}}, v_{x_{l}}$ and $q_{x_{i} x_{j}}$. Let $L_{2}^{(0)}$ be the operator whose coefficients of $L_{2}$ at the origin. Then by a coordinate transformation we get

$$
\int q\left(\sum_{i, j} v_{x_{i} x_{i}}^{2}\right) d x \leqq \int q\left(L_{2}^{(0)} v\right)^{2} d x+\int F\left(q_{x_{i} z}, v_{x_{k}}\right) d x
$$

Obviously

$$
\begin{aligned}
& \int q\left(L_{2}^{(0)} v\right)^{2} d x \leqq c\left(\int q\left(L_{2} v\right)^{2} d x+\int q\left(\left(L_{2}^{(0)}-L_{2}\right) v\right)^{2} d x\right) \\
& \leqq c\left(\int q\left(L_{2} v\right)^{2} d x+d \int q\left(\sum_{i, j} v_{x_{i x}}^{2}\right) d x\right) .
\end{aligned}
$$

Thus if we take $d$ sufficiently small, (6.4) becomes

$$
\int q\left(\sum_{i, j} v_{x_{x} x_{j}}^{2}\right) d x \leqq c\left(\int q\left(L_{2} v\right)^{2} d x+\int F\left(q_{x_{i} x_{j}}, v_{x_{k}}\right) d x\right) .
$$

Hence we have

$$
\int q\left(\sum_{i, j, l} v_{x_{i} x_{j} x_{l}}^{2}\right) d x \leqq c\left(\int q\left(L_{2} v_{x_{l}}\right)^{2} d x+\int F\left(q_{x_{x_{x}},}, v_{x_{l} x_{k}}\right) d x\right) .
$$

Now it is seen

$$
\left|L_{2} v_{x_{l}}\right| \leqq\left|\left(L_{2} v\right)_{x_{l}}\right|+\left|L_{2} v_{x_{i}}-\left(L_{2} v\right)_{x_{l}}\right| \leqq\left|\left(L_{2} v\right)_{x_{l}}\right|+c \sum_{i, j}\left|v_{x_{i} x_{j}}\right|
$$

Therefore we obtain from (6.6) 
(6.8)

$$
\begin{aligned}
\int q\left(\sum_{i, j, l} v_{x_{t} x_{j} x_{l}}^{2}\right) d x \leqq c\left[\int q\left(\left(L_{2} v\right)_{x_{l}}\right)^{2} d x\right. \\
\left.+\sum_{i, j} \int q v_{\lambda_{l} x_{j}}^{2}+\sum_{i, j, k, l} \int\left|q_{x_{t},}\right| v_{\lambda_{\xi} x_{l}}^{2} d x\right] .
\end{aligned}
$$

Let us estimate the following from the above:

$$
\int r^{4} \Phi^{1-\delta} \exp \left(2 n \Phi ^ { \delta } \left(\left(I_{2} v\right)^{2} d x\right.\right.
$$

The integral can be rewritten in the form

$$
\int r^{-4} \bar{\Phi}^{2 \delta-3} \exp \left(2 n \bar{\phi}^{\delta}\right)\left(r^{4} \bar{\Phi}^{2-\frac{3}{2} 5} L_{2} v\right)^{2} d x
$$

By Proposition 5.2 we see

$$
\begin{aligned}
& n^{3} \int r^{-4} \Phi^{2 \delta-3} \exp \left(2 n \Phi^{\delta}\right)\left(r^{4} \Phi^{2-\frac{3}{2} \delta} L_{2} v\right)^{2} d x \\
& \quad+n \int \Phi^{-1} \exp \left(2 n \Phi^{\delta}\right)\left|\nabla\left(r^{-1} \Phi^{2-\frac{3}{2} \delta} L_{2} v\right)\right|^{2} a^{7} x \\
& \leqq c \int r^{4} \Phi^{1-\delta} \exp ^{\operatorname{x}}\left(2 n \Phi^{\delta}\right)\left(L_{1}\left(r^{4} \Phi^{2-\frac{3}{2} \delta} L_{2} v\right)\right)^{2} d x
\end{aligned}
$$

An easy computation shows

$$
\begin{aligned}
& \left|\nabla\left(r^{4} \Phi^{2-\frac{3}{2} \delta}\right)\right| \leqq c r^{2}{\Phi^{1-\frac{3}{2}} \delta}^{-3} \\
& \left|\left(\gamma^{4} \bar{\Phi}^{2-\frac{3}{2} \hat{\delta}}\right)_{x_{2} x_{j}}\right| \leqq c \Phi^{-\frac{3}{2} \delta}
\end{aligned}
$$

Thus taking $n$ as sufficiently large (or $d$ as sufficiently small) in (6.9), we have

$$
\begin{aligned}
& n^{3} \int r^{4} \Phi^{1-\delta} \exp \left(2 n \Phi^{\delta}\right)\left(L_{2} v\right)^{2} d x \\
& \quad+n \int r^{8}{\Phi^{3-3 \delta}}^{2} \exp \left(2 n \Phi^{\delta}\right)\left|\nabla\left(L_{2} v\right)\right|^{2} d x \\
& \leqq c\left\{\int r^{12} \Phi^{5-4 \delta} \exp \left(2 n \Phi^{\delta}\right)\left(L_{1} L_{2} v\right)^{2} d x\right. \\
& \quad+\int r^{8} \Phi^{3-4 \delta} \exp \left(2 n \Phi^{\delta}\right)\left|\nabla\left(L_{2} v\right)\right|^{2} d x \\
&\left.\quad+\int r^{1} \Phi^{1-4 \delta} \exp \left(2 n \Phi^{\delta}\right)\left(L_{2} v\right)^{2} d x\right\}
\end{aligned}
$$

Hence we obtain 


$$
\begin{aligned}
& n^{3} \int r^{4} \Phi^{1-\delta} \exp \left(2 n \Phi^{\delta}\right)\left(L_{2} v\right)^{2} d x \\
& \quad+n \int r^{8} \Phi^{3-3 \delta} \exp \left(2 n \Phi^{\delta}\right)\left|\nabla\left(L_{2} v\right)\right|^{2} d x \\
& \leqq c \int r^{12} \Phi^{5-4 \delta} \exp \left(2 n \bar{\Phi}^{\delta}\right)\left(L_{1} L_{2} v\right)^{2} d x .
\end{aligned}
$$

Combining Proposition 5.2 and (6.10), we see

$$
\begin{aligned}
& \int r^{-4} \bar{\Phi}^{2 \delta-3} \exp \left(2 n \Phi^{\delta}\right) v^{2} d x \\
& +n^{-2} \int \bar{\Phi}^{-1} \exp \left(2 n \Phi^{\delta}\right)|\nabla v|^{2} d x
\end{aligned}
$$

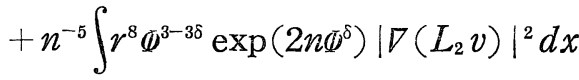

$$
\begin{aligned}
& \leqq c n^{-6} \int r^{12} \Phi^{5-4 \delta} \exp \left(2 n \Phi^{\delta}\right)\left(L_{1} L_{2} v\right)^{2} d x \text {. }
\end{aligned}
$$

We set in $(6.5)$

$$
q=n^{-4} r^{8} \Phi^{3-2 \hat{o}} \exp \left(2 n \Phi^{\delta}\right) .
$$

Then it is easily seen that

$$
\left|q_{x_{i} x_{j}}\right| \leqq n^{-2} r^{4} \Phi \exp \left(2 n \Phi^{\delta}\right)
$$

Hence for a sufficiently small $d$ we have by (6.5), (6.10) and (6.11)

$$
\begin{aligned}
& \int r^{-4} \Phi^{2 \delta-3} \exp \left(2 n \Phi^{\delta}\right) v^{2} d x \\
& +n^{-2} \int \Phi^{-1} \exp \left(2 n \Phi^{\delta}\right)|\nabla v|^{2} d x \\
& +n^{-4} \int r^{8} \Phi^{3-2 \delta} \exp \left(2 n \Phi^{\delta}\right)\left(\sum v_{x_{i} x_{j}}^{2}\right) d x \\
& \leqq c n^{-6} \int r^{12} \bar{\Phi}^{5-4 \delta} \exp \left(2 n \Phi^{\delta}\right)\left(L_{1} L_{2} v\right)^{2} d x \text {. }
\end{aligned}
$$

Secondly we set in (6.8)

$$
q=n^{-6} r^{12} \bar{\phi}^{5-4 \delta} \exp \left(2 n \Phi^{\delta}\right)
$$

Then we easily see

$$
\left|q_{x_{i}, x}\right| \leqq c n^{-1} r^{8} \Phi^{3-2 \delta} \exp \left(2 n \Phi^{\delta}\right) .
$$

Thus combining $(6.8),(6.5),(6.11)$ and $(6.12)$, we obtain the estimate (6.1). 
7. Now we shall prove Theorem 1 and 2 . First we shall prove Theorem 2.

Proof of Theorem 2. We may assume that $\Gamma=\Omega_{a}$.

Let $\delta$ and $d_{0}$ be the positive numbers in Proposition 6.1. Let the assumption on $u$ be such that

$$
D^{\alpha} u=o\left(\exp \left(-r^{-2 \delta-\varepsilon}\right)\right) \quad(r \rightarrow 0, \varepsilon>0,|\alpha| \leqq 3)
$$

along $\Gamma$. Then by Proposition 4.1 we see

$$
D^{\alpha} u=o\left(\exp \left(-r^{-\delta-(\varepsilon / 3)}\right)\right) \quad(r \rightarrow 0,|\alpha| \leqq 3) \text { in } S_{1 / 2} \cap \Omega_{a} .
$$

Now we take a $C^{\infty}$ function $\eta(t)$ such that

$$
\eta(t)= \begin{cases}1 & 0 \leqq t \leqq d_{0} / 2 \\ 0 & t \geqq d_{0} .\end{cases}
$$

And put $\zeta(x)=\eta\left(r^{2} / x_{1}\right)$. Then we easily see

$$
D^{k}\left(r^{2} / x_{1}\right)=D^{k}\left(x_{1}^{-1} r^{2}\right) \leqq c r^{-2(k+1)} \text {. }
$$

Hence it holds

$$
\left|D^{k} \zeta\right| \leqq c_{k} r^{-c_{k}}
$$

where $c_{k}$ is a constant depending on $k$. Set $v(x)=\zeta(x) u(x)$. We note that $v=u$ in $S_{d_{0} / 2}$. Then by (7.1) and (7.2) it is seen that $v \in \mathscr{F}_{4, \delta}\left(S_{d_{0}}\right)$. We can assume that the constant on the right of (1.4) is sufficiently small. Applying Proposition 6.1 to $v$, we have from (1.4)

$$
\begin{gathered}
c \int_{S_{d_{0}}-S_{d_{0} / 2}} r^{12} \Phi^{5-4 \delta} \exp \left(2 n \Phi^{\delta}\right)\left(L_{1} L_{2} v\right)^{2} d x \\
\geqq n^{6} \int r^{-4} \Phi^{2 \delta-3} \exp \left(2 n \Phi^{\delta}\right) u^{2} d x .
\end{gathered}
$$

Here we note that for some positive constant $c$

$$
r^{12} \Phi^{5-4 \delta} \leqq c \leqq r^{-4} \Phi^{2 \delta-3}
$$

and

$$
\begin{aligned}
& \Phi \geqq 2 / d_{0} \text { in } S_{d_{0} / 2} \\
& \Phi \leqq 2 / d_{0} \text { in } S_{d_{0}}-S_{d_{0} / 2} .
\end{aligned}
$$

Hence (7.3) is reduced to 


$$
c n^{-6} \int_{S_{d_{0}}-S_{d_{0} / 2}}\left(L_{1} L_{2} v\right)^{2} d x \geqq \int_{S_{d_{0} / 2}} u^{2} d x
$$

Let $u$ tend to infinity. Then $u=0$ in $S_{d_{0} / 2}$. Therefore $u$ vanishes identically by the well known result with respect to the uniqueness in Cauchy's problem.

Proof of Theorem 1. It is sufficient to prove the existence of $\delta$ such that the solutions $\left\{u_{p}\right\}$ of (3.15) satisfying

$$
u_{p} \in C^{2}(\bar{\Omega}) \cap C^{3}(\Gamma)
$$

and

$$
\begin{gathered}
u_{p}, u_{p x_{i}}=o\left(\exp \left(-r^{-2 \delta-\varepsilon}\right)\right) \quad(r \rightarrow 0, \varepsilon>0) \text { along } \Gamma \\
(p=1, \cdots, m, i=1, \cdots, N)
\end{gathered}
$$

vanish identically in $\Omega$.

We obtain from Proposition 5.2

$$
\begin{aligned}
& c \int r^{4} \Phi^{1-\delta} \exp \left(2 n \bar{\Phi}^{\varsigma}\right)\left(L_{p} v_{p}\right)^{2} d x \\
& \geqq n^{3} \int r^{-4} \Phi^{2 \delta^{-3}} \exp \left(2 n \Phi^{\delta}\right) v_{p}^{2} d x \\
& \quad+n \int \Phi^{-1} \exp \left(2 n \Phi^{\delta}\right)\left|\nabla v_{p}\right|^{2} d x .
\end{aligned}
$$

Set $v_{p}(x)=\zeta(x) u_{p}$ for $\zeta$ in (7.1) and for the solutions $u_{p}$. Then by Proposition 4.2, we see $v_{p} \in \mathscr{F}_{2, \delta}\left(S_{d_{0}}\right)$. Summing up (7.6) with respect to $p$ and noting that $u_{p}$ are solutions of (3.15), we see in a similar way to that in the proof of Theorem 2

$$
c n^{-3} \int_{S_{d_{0}}-S_{d_{0} / 2}} \sum_{p=1}^{m}\left(L_{p} v_{p}\right)^{2} d x \geqq \sum_{p=1}^{m} \int_{S_{d_{0} / 2}} u_{p}^{2} d x_{0}
$$

Thus tending $\imath$ to infinity, we have completed the proof.

Finally we give some corollaries.

Carollary 7.1. Let $u$ be a solution in $\bar{\Omega}_{a}$ of

$$
|\operatorname{Lu}| \leqq C\left(x_{1}^{-2}|u|+x_{1}^{-1} \sum_{i=1}^{n}\left|u_{x_{i}}\right|\right),
$$

where $L$ is an operator of the form (1.1). Then there is a positive number $\delta$ depending only on $L$ such that if 


$$
u, D u=o\left(\exp \left(-r^{-\delta}\right)\right) \quad(r \rightarrow 0) \text { in } S_{1 / 2} \cap \bar{\Omega}_{a},
$$

then u vanishes identically.

Proof. Wre proceed in a similar manner to that in the proof of Theorem 1. Put $v(x)=\zeta(x) u(x)$ for $\zeta$ in (7.2). Then we have

$$
\begin{aligned}
& \int_{S_{d_{0}}} r^{4} \Phi^{1-\delta} \exp \left(2 n \Phi^{\S}\right)(L v)^{2} d x \\
& \leqq c \int_{S_{i_{0} / 2}} r^{4} \Phi^{1-\delta} \exp \left(2 n \Phi^{\delta}\right)\left(x_{i}^{-4}|u|^{2}+x_{1}^{-2} \sum_{i=1}^{N}\left|u_{x_{i}}\right|^{2}\right) d x \\
& +c \int_{S_{d_{0}}-S_{d_{0} / 2}} r^{4} \bar{\phi}^{1-\delta} \exp \left(2 n n \bar{\phi}^{\hat{\delta}}\right)(L v)^{2} d x
\end{aligned}
$$

The first integral on the right of this inequality is estimated from the above by

$$
\begin{aligned}
& c\left(\int_{S_{d_{0} / 2}} r^{-4} \Phi^{1-\delta} \exp \left(2 n \phi^{\delta}\right)|u|^{2} d x\right. \\
& \quad+\int_{S_{d_{0} / 2}} \Phi^{1-\delta} \exp \left(2 n \Phi^{\delta}\right)|\nabla u|^{2} d x .
\end{aligned}
$$

Thus combining this inequality with Proposition 5.2, we obtain

$$
\begin{gathered}
c n^{-3} \int_{S_{d_{0}}-S_{d_{0} / 2}} r^{4} \bar{\phi}^{1-\varepsilon} \exp \left(2 n \bar{\phi}^{5}\right)(I v)^{2} d x \\
\geqq \int_{S_{i_{0} / 2} \gamma^{-4} \Phi^{2 \delta-3} \exp \left(2 n \Phi^{5}\right) u^{2} d x}
\end{gathered}
$$

Hence we complete the proof in the same way as in Theo:ern 1.

Similariy to Corollary 7.1 we can easily prove the foilowing

Corollary 7.2. Let $u$ be a solution in $\bar{\Omega}_{a}$ of

$$
\left|L_{1} L_{2} u\right| \leqq c \sum_{|\alpha| \leqq 3} x_{1}^{|\alpha|-3}\left|D^{\alpha} u_{i}\right|
$$

where $I_{1}$ and $L_{2}$ are of the form (1.1) whose coefficienis are sufficiently smooth. Than there is a positive number $\delta$ depending only on $L_{1}$ and $L_{2}$ such that if for $|\alpha| \leqq 3$

$$
D^{\alpha} u=o\left(\operatorname{erp}\left(-r^{-\delta}\right)\right) \quad(r \rightarrow 0) \text { in } S_{1 / 2} \cap \Omega_{a},
$$

then $u$ vanishes identically. 


\section{References}

[1] Aronszajn, N., A unique continuation theorem for solutions of elliptic partial differential equations or inequalities of second order, J. Math. Pures Appl. 36 (1957), 235-249.

[2] Bernstein, I. S., On the unique continuation problem of elliptic partial differential equations, J. Math. \& Mech. 10 (1961), 579-606.

[3] Calderón, A. P., Uniqueness in the Cauchy problem for partial differential equations, Amer. J. Math. 80 (1958), 16-36.

[4] Carleman, $T$, Sur un problème d'unicité pour les systèmes d'équations aux dériveés partiells à deux variables indépendants, Arkiv Mat. 26 B (1938), 1-9

[5] Cordes, H. O., Über die Bestimmtheit der Lösungen elliptischer Differentialgleichungen durch Anfangsvorgaben, Nachr. Akad. Wiss. Göttingen, 11 (1956), 239-258.

[6] Douglis, A., On uniqueness in Cauchy problems for elliptic systems of equations, Comm. Pure Appl. Math. 13 (1960), 593-607.

[7] Hartman, P. and A. Wintner, On the local behavior of solutions of non-parabolic partial differential equations, III. Approximations by spherical harmonics, Amer. J. Math. 77 (1955), 453-483.

[8] Hayashida, K., On the uniqueness in Cauchy's problem for elliptic equations, RIMS Kyoto Univ. Ser. A, 2 (1967), 429-449.

[9] Heinz. E., Über die Eindeutigkeit beim Cauchyschen Anfangswert-problem einer elliptischen Differentialgleichung zweiter Ordnung, Nachr. Akad. Wiss. Göttingen, $\mathbb{1}$ (1955), 1-12.

[10] Hörmander, L., On the uniqueness of the Cauchy problem I-II, Math. Scand. 6 (1958), 213-225; 7 (1959), 177-190.

[11] Kumano-Go, H., Unique continuation for elliptic equations, Osaka Math. J. 15 (1963), 151-172.

[12] Landis, E. M., On some properties of elliptic equations, Dokl. Akad. Nauk SSSR, 107 (1956), 640-643 (Russian).

[13] Lavrentév, M. M., On Cauchy's boundary value problem for linear elliptic equations of the second order, Dokl. Akad. Nauk SSSR, 112 (1957), 195-197 (Russian).

[14] Mergelyan, S. N., Harmonic approximation and approximate solution of Cauchy's problem for Laplace equation, Dokl. Akad. Nauk SSSR, 107 (1956), 644-647 (Russian).

[15] Mizohata, S., Unicité du prolongement des solutions des équation elliptiques du quatrième ordre, Proc. Japan Acad. 34 (1958), 687-692.

[16] Müller, C., On the behavior of solutions of the differential equations $\Delta U=F(x . U)$ in the neighborhood of a point, Comm. Pure Appl. Math. 7 (1954), 505-516.

[17] Pederson, R. N., On the unique continuation theorem for certain second and fourth order elliptic equations, Comm. Pure Appl. Math. 9 (1958), 67-80.

[18] Protter, M H., Unique continuation for elliptic equations, Trans. Amer. Math. Soc. 95 (1960), 81-91.

[19] Shirota, T., A remark on the unique continuation theorem for certain fourth order elliptic equations, Proc. Japan Acad. 36 (1960), 571-573. 